\title{
Long-term outcomes following surgical repair of coronary artery fistula in adults
}

\author{
Yuki Wada ${ }^{1}$, Akira Marui ${ }^{1}$, Yoshio Arai $^{1}$, Atsushi Nagasawa ${ }^{1}$, Shinichi Tsumaru ${ }^{1}$, Ryoko \\ Arakaki $^{1}$, Jun Iida ${ }^{1}$, Yuki Kuroda ${ }^{1}$, Yumeka Tamai ${ }^{1}$, Takashi Fukushima ${ }^{1}$, and Yoshiharu \\ Soga ${ }^{1}$
}

\author{
${ }^{1}$ Kokura Memorial Hospital
}

July 9, 2021

\begin{abstract}
Background and aim of the study: Coronary artery fistula (CAF) is a relatively rare cardiac anomaly. We investigated longterm outcomes following surgical repair of CAF in adults. Methods: We retrospectively investigated 13 consecutive patients undergoing surgical repair of CAF in our institution between 2008 and 2019 (67.3 \pm 10.4 years old, $38 \%$ male). CAF types were coronary artery-pulmonary artery fistula (77\%), coronary artery-coronary sinus fistula (15\%), and both (8\%). CAFs originated from the left coronary artery $(38 \%)$, right coronary artery $(8 \%)$, and bilateral coronary arteries $(38 \%)$. Pulmonary and systemic flow (Qp/Qs) was measured in seven patients (54\%), with a mean value of 1.52 . Seven patients underwent surgery for CAFs alone, and others simultaneously underwent surgery for comorbid cardiac diseases. Results: All procedures were conducted under cardiopulmonary bypass. Surgical procedures were direct epicardial ligation of fistula (92\%), direct closure of CAF through pulmonary artery incision (38\%), direct closure of CAF through coronary sinus incision (8\%), or patch closure of CAF through coronary artery incision (8\%). Myocardial perfusion scintigraphy showed asymptomatic myocardial ischemia in the right coronary area after surgery in one patient. There were no deaths perioperatively or during follow-up (mean 66.6 months). There were no coronary or other CAF-related events. Conclusions: Several anatomical variations in CAF were observed which coexist with cardiac disease. Long-term outcomes following surgical repair were satisfactory, and the concurrent intervention of CAFs during surgery for comorbid cardiac disease is useful to prevent future complications related CAFs in adults.
\end{abstract}

\section{Introduction}

A coronary arteriovenous fistula $(\mathrm{CAF})$ is a rare anomaly caused by abnormal communication between a coronary artery and any cardiac cavity or great vessel. ${ }^{1}$ While some cases are asymptomatic and found incidentally, others are associated with symptoms including heart failure and myocardial ischemia. ${ }^{2,3,4}$ Symptomatic cases such as those with coronary stealing syndrome and heart failure owing to shunting are indicated for surgery, while asymptomatic cases with anatomical findings such as aneurysm formation also require surgery. ${ }^{4,5,6}$ Although some studies have reported surgical techniques for CAFs, ${ }^{7,8}$ few have reported long-term outcomes following surgical repair of CAFs in adults. Herein, we report the clinical characteristics, surgical procedures, and long-term outcomes of 13 patients with CAFs.

\section{Materials and methods}

A total of 13 patients underwent surgical treatment for CAFs in our hospital from 2008 to 2019. There were five men (38\%), and the mean age was 67.3 years (45-81 years). The CAF was assessed by preoperative coronary artery computed tomography $(\mathrm{CT})$. Coronary angiography $(\mathrm{CAG})$ was performed in 11 patients (85\%), and six patients (46\%) underwent right heart catheterization. This study was approved by the Ethical Committee in Kokura Memorial Hospital (ID: 20061501, date 2020/06/05). Written informed consent was obtained from the patients for publication of this case report and accompanying images. 


\section{Results}

Of the 13 cases, 10 (77\%) had coronary artery-pulmonary artery fistula (CA-PA fistula) and two (15\%) had coronary artery-coronary sinus fistula (CA-CS fistula) (Table 1). There was also one case (8\%) with CA-PA fistula and one with CA-CS fistula. Seven patients (54\%) had the following comorbid cardiovascular diseases: aortic stenosis, aortic regurgitation, mitral regurgitation, tricuspid regurgitation, ascending aortic dilatation, and patent foramen ovale. Pulmonary and systemic flow (Qp/Qs) was measured in seven cases (54\%), and its average was 1.52 (Table 1). A majority of the CAFs with arterial origins were right coronary artery (RCA) or left coronary artery (LCA); however, they also originated from other regions, such as the aorta and left internal thoracic artery in a small number of cases. The majority of the coronary artery fistulas drained into the main pulmonary artery (Table 2). The formation of an aneurysm in abnormal blood vessels was found in three cases (23\%), and one of these caused its rupture. Coronary sinus dilatation was observed in all cases with CA-CS fistula. Seven patients (54\%) underwent surgery for CAFs alone, and others simultaneously underwent surgery for comorbid cardiac diseases.

Preoperative ECG findings were as follows: normal sinus rhythm $(\mathrm{n}=9,69 \%)$, atrial fibrillation $(\mathrm{n}=2,15 \%)$, multifocal supraventricular extrasystole $(\mathrm{n}=1,8 \%)$, left atrial enlargement $(\mathrm{n}=1,8 \%)$. Preoperative transthoracic echocardiography showed no asynergy in any of the cases and low $\mathrm{EF}\left(\mathrm{EF}_{i} 50 \%\right)$ in four patients (30\%). Myocardial scintigraphy was performed in two cases $(15 \%)$, one of which showed small regional ischemia in the peripheral region of the RCA.

Operations were performed via median sternotomy and through the use of the cardiopulmonary bypass in all patients. The surgical procedure for CAFs was as follows: direct epicardial ligation of CAFs alone ( $\mathrm{n}=6,46 \%)$, direct epicardial ligation of CAFs plus direct closure of CAFs through pulmonary artery incision $(\mathrm{n}=5,38 \%)$, direct closure of CAF through coronary sinus incision $(\mathrm{n}=1,8 \%)$, patch closure of CAF through coronary artery incision $(\mathrm{n}=1,8 \%)$ (Table 3$)$. Concomitant procedures were Aortic Valve Replacement (AVR) ( $\mathrm{n}=4$, 15\%), Mitral Valve Replacement (MVP) ( $\mathrm{n}=2,15 \%)$, Tricuspid Valve Annuloplasty (TAP) (n=2, 15\%), and others $(\mathrm{n}=2,15 \%)$.

There were no operative deaths and no significant ST-T change in ECG after surgery. One patient showed asymptomatic myocardial ischemia postoperatively. Moreover, poor contrast enhancement of RCA \#2 on coronary $\mathrm{CT}$ for was found in routine postoperative examination (Figure 1). Myocardial perfusion scintigraphy showed mild regional ischemia. No ECG and TTE changes were observed in this case compared with preoperative data. The patient was asymptomatic and monitored with anticoagulation therapy.

The average postoperative follow-up period was 66.2 months (11-138 months) without any late deaths. During follow-up, one patient $(8 \%)$ underwent thoracic aortic surgery (Bentall procedure and total arch replacement) two years after primary surgery. Another patient presented with paroxysmal atrial tachycardia nine years after surgery. There were no CAF-related events, including myocardial ischemia.

\section{Discussion}

Coronary artery fistula is a condition with direct communication between the coronary artery and the large vessels or the atria/ventricle by abnormal blood vessels without passing through the capillary beds. CAFs are found in $0.18 \%$ of patients who have undergone coronary angiography ${ }^{1}$. In previous research, most CAFs have been found to originate from the RCA or LCA. ${ }^{9,10,11}$ Five cases at our hospital showed RCA origin, and a further five originated from both the RCA and LCA. The right heart system is known to be the most common perfusion site for CAFs. ${ }^{9,10,11,12}$. In our cohort, 10 (77\%) cases drained into the PA, two (15\%) into the CS, and one (8\%) into the PA \& CS. All cases drained into the right heart system.

CAFs are indicated for surgery when associated with symptoms, such as coronary steal phenomenon due to fistula, myocardial ischemia caused by increased shunt blood flow, heart failure, arrhythmia, cardiac dilatation, and infective endocarditis. ${ }^{2,4}$ Furthermore, due to reports of ruptured aneurysms, ${ }^{3}$ cases that develop an aneurysm, though asymptomatic, are also considered for treatment. Surgical indication for asymptomatic cases without an aneurysm, however, is controversial. Some literature recommends surgery for coronary ar- 
teriovenous fistulas during diagnosis to prevent future coronary artery disease and infective endocarditis. ${ }^{4,5,6}$ However, surgery does not lead to long-term changes in some cases, and follow-up observation has been recommended. ${ }^{13}$ Seven cases $(54 \%)$ at our hospital were indicated for surgery owing to CAFs (dyspnea on exertion or aneurysm formation). However, six cases (46\%) were indicated for surgery owing to comorbid cardiac disease. The concurrent intervention of CAFs during surgery of comorbid cardiac disease is considered useful in preventing future complications related to CAFs.

For CAF surgery, abnormal blood vessels are treated with procedures including ligation of the abnormal blood vessel from the epicardial side, direct closure of the fistula, and indirect closure of the fistula from the epicardium. ${ }^{7,12}$ Surgical methods also vary depending on the use of other techniques, including the cardiopulmonary bypass and the coronary artery bypass graft surgery. ${ }^{7,14}$ In principle, the resection of an aneurysm is necessary due to its reported rupture risks. ${ }^{3}$ At our hospital, cardiopulmonary bypass was used in all cases.

Perioperative death has been previously reported in some CAF cases, with myocardial ischemia and arrhythmia presenting as critical perioperative complications. ${ }^{7,8,9}$ Said et al. reported that perioperative myocardial ischemia was an important perioperative complication. ${ }^{7}$ Risk factors in early and late postoperative periods include large fistula formation, coronary artery dilation, and aneurysm formation. Myocardial ischemia in the early postoperative period is caused by the occlusion of critical branches during the treatment of a fistula. Conversely, myocardial ischemia in the late postoperative period is caused by thrombus formation in coronary arteries. To prevent myocardial ischemia in the early postoperative period, Cheung et al. performed the temporary clamping of a fistula before its ligation during surgery and monitored changes in hemodynamics and ischemia. ${ }^{14}$ The wall motion after ligation was assessed by transesophageal echocardiography. Revascularization, such as coronary artery bypass graft surgery, is considered when myocardial ischemia is indicated by clinical findings. An approach to treating myocardial ischemia in the late postoperative period is the use of warfarin to prevent thrombus formation. ${ }^{14}$

Although no perioperative deaths were observed at our hospital, one case (8\%) showed poor contrast RCA\#24 on postoperative coronary CT. In this case, myocardial scintigraphy showed small ischemia in the region supplied by the RCA, while postoperative myocardial scintigraphy showed no significant change compared to the preoperative state. Revascularization was not performed because asynergy was not observed either pre- or peri-operatively. This case was asymptomatic and showed no postoperative asynergy; therefore, we followed up with oral administration of aspirin and warfarin.

\section{Conclusion}

Several anatomical variations were observed in CAF, and they were found to coexist with cardiac disease. Long-term outcomes following surgical repair were satisfactory, and the concurrent intervention of CAFs during surgery of comorbid cardiac disease is useful in preventing future complications related to CAFs in adults.

\section{Acknowledgments: none}

\section{Author contributions statement}

Yuki Wada: Writing - original draft; Writing - review \& editing

Akira Marui: Conceptualization

Yoshio Arai: Investigation

Atsushi Nagasawa: Investigation

Shinichi Tsumaru: Investigation

Ryoko Arakaki: Investigation

Jun Iida: Investigation 
Yuki Kuroda: Investigation

Yumeka Tamai: Investigation

Takashi Fukushima: Investigation

Yoshiharu Soga: Superivision

\section{References}

1. Yamanaka O, Hobbs RE. Coronary artery anomalies in 126,595 patients undergoing coronary arteriography. Catheter Cardio Inte 1990;21:28-40.

2. Warnes CA, Williams RG, Bashore TM, Child JS, Connolly HM, Dearani JA, et al. ACC/AHA 2008 guidelines for the management of adults with congenital heart disease: A report of the American College of Cardiology/American Heart Association Task Force on Practice Guidelines (Writing Committee to Develop Guidelines on the Management of Adults With Congenital Heart Disease). Developed in Collaboration With the American Society of Echocardiography, Heart Rhythm Society, International Society for Adult Congenital Heart Disease, Society for Cardiovascular Angiography and Interventions, and Society of Thoracic Surgeons. J Am Coll Cardiol 2008;52:e143-e263.

3. Kanki S, Kakita M, Woo E, Sasaki T, Daimon M, Katsumata T. Ruptured coronary aneurysm with a congenital coronary arteriovenous fistula. Jpn J Cardiovasc Surg 2015;44:198-202.

4. Oldham HN Jr, Ebert PA, Young WG, Sabiston DC Jr. Surgical management of congenital coronary artery fistula. Ann Thorac Surg 1971;12:503-513.

5. Urrutia-S CO, Falaschi G, Ott DA, Cooley DA. Surgical management of 56 patients with congenital coronary artery fistulas. Ann Thorac Surg 1983;35:300-307.

6. Liberthson RR, Sagar K, Berkoben JP, Weintraub RM, Levine FH. Congenital coronary arteriovenous fistula: Report of 13 patients, review of the literature and delineation of management. Circulation 1979;59:849-853.

7. Said SM, Burkhart HM, Schaff HV, Connolly HM, Phillips SD, Suri RM, et al. Late outcome of repair of congenital coronary artery fistulas-a word of caution. J Thorac Cardiovasc Surg 2013;145:455-460.

8. Armsby LR, Keane JF, Sherwood MC, Forbess JM, Perry SB, Lock JE. Management of coronary artery fistulae: Patient selection and result of trancatheter closure. J Am Coll Cardiol 2002;39:1026-32.

9. Challoumas D, Pericleous A, Dimitrakaki IA, Danelatos C, Dimitrakakis G. Coronary arteriovenous fistula: A Review. Int J Angiol 2014;23:1-10.

10. Zhou K, Kong L, Wang Y, Li S, Song L, Wang Z, et al. Coronary artery fistula in adults: Evaluation with dual source CT coronary angiography. Br J Radiol 2015;88:20140754.

11. Fernades ED, Kadivar H, Hallman GL, Reul GJ, Ott DA, Cooley DA. Congenital malformations of the coronary arteries: The Texas Heart Institute Experience. Ann Thorac Surg 1992;54:732-740.Reul RM, Cooley, DA, Hallman GL, Reul GJ. Surgical treatment of coronary artery anomalies: Report of a $37 \frac{1}{2}$-year experience at the Texas Heart Institute. Tex Heart Inst J 2002;29:299-307.

12. Angelini P. Coronary artery anomalies: An entity in search of an identity. Circulation. 2007;115:12961305

13. Ata Y, Turk T, Bicer M, Yalcin M, Ata F, Yavuz S. Coronary arteriovenous fistulas in the adults: Natural history and management strategies. J Cardiothorac Surg 2009;4:62.

14. Cheung DL, Au WL, Cheung HH, Chiu CS, Lee WT. Coronary artery fistulas: Long-term results of surgical correction. Ann Thorac Surg 2001;71:190-195.

\section{Tables}

Table 1 . Patient background

\begin{tabular}{ll}
\hline & $\mathrm{n}(\%)$ \\
\hline Number of cases & 13 \\
Age & $67.3 \pm 10.4$
\end{tabular}




\begin{tabular}{ll}
\hline & $\mathrm{n}(\%)$ \\
\hline Male & $5(38)$ \\
Mean follow-up (month) & $66.6(11-138)$ \\
CAFs type & \\
CA-PA fistula & $10(77)$ \\
CA-CS fistula & $2(15)$ \\
CA-PA fistula + CA-CS fistula & $1(8)$ \\
Qp/Qs (evaluated in 7 cases) & $1.52 \pm 0.49$ \\
Preoperative ECG & $9(69)$ \\
Normal sinus rhythm & $2(15)$ \\
Atrial fibrillation & $1(8)$ \\
Multifocal supraventricular extrasystole & $1(8)$ \\
Left atrial enlargement & $4(30)$ \\
Low EF (EF;50\%) & \\
Comorbid cardiac disease & $2(15)$ \\
MR & $1(8)$ \\
AS & $1(8)$ \\
TR & $1(8)$ \\
AR + MR+ TR & $1(8)$ \\
AR + Dilation of ascending aorta & $1(8)$ \\
AR + Dilation of ascending aorta + PFO & \\
Surgical indication & $7(54)$ \\
CAFs & $6(46)$ \\
Comorbid cardiac disease &
\end{tabular}

AR, aortic regurgitation; AS, aortic stenosis; CA, coronary artery; CAF, coronary artery fistula; CS, coronary sinus; MR, mitral regurgitation; PA, pulmonary artery; PFO, patent foramen oval; TR, tricuspid regurgitation;

Table 2 . Sites of origin and drainage of coronary artery fistulas / aneurysm formation

\begin{tabular}{ll}
\hline & $\mathrm{n}(\%)$ \\
Site of origin & $5(38)$ \\
LCA & $5(38)$ \\
LCA + RCA & $1(8)$ \\
RCA & $1(8)$ \\
RCA + LCA + Aortic arch & $1(8)$ \\
RCA + LCA + LITA + Aortic arch & \\
Drainage site & $10(77)$ \\
PA & $2(15)$ \\
CS & $1(8)$ \\
PA + CS & \\
Aneurysm formation & $2(15)$ \\
Unruptured & $1(8)$ \\
Ruptured & \\
\hline
\end{tabular}

CS, coronary sinus; LCA, left coronary artery; LITA, left internal thoracic artery; PA, pulmonary artery; $\mathrm{RCA}$, right coronary artery

Table 3 . Surgical procedure for CAFs 


\begin{tabular}{ll}
\hline & $\mathrm{n}(\%)$ \\
\hline Median sternotomy & $13(100)$ \\
Cardiopulmonary bypass & $13(100)$ \\
Techniques for CAFs & \\
Direct epicardial ligation & $6(46)$ \\
Direct epicardial ligation + Direct closure through PA incision & $5(38)$ \\
Direct closure through CS incision & $1(8)$ \\
Patch closure through CS incision & $1(8)$ \\
\hline
\end{tabular}

CAFs, coronary artery fistula; CS, coronary sinus; PA, pulmonary artery

\section{Figure Legend}

Figure 1. Poor contrast enhancement of the RCA\#2 on coronary CT was found during routine postoperative examination.
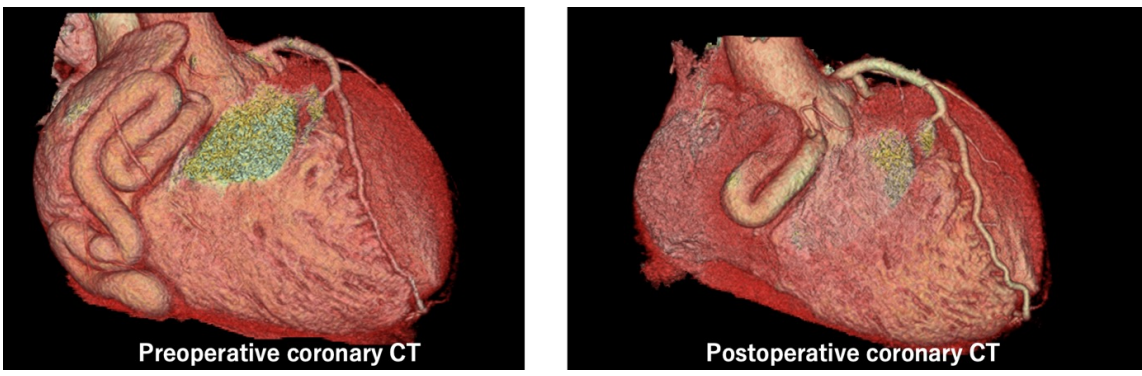